\title{
The conceptual design of a complex technical object based on intelligent technologies
}

\author{
R R Karimov ${ }^{1}$, N V Kondratyeva ${ }^{2}$, E A Kuzmina ${ }^{1}$, A S Kovtunenko ${ }^{1}$, M A \\ Verkhoturov $^{1}$, and S S Valeev ${ }^{1,2}$ \\ ${ }^{1}$ Ufa State Aviation Technical University, K. Marx Str. 12, Ufa, 450008, Russia \\ ${ }^{2}$ Sochi State University, Politekhnicheskaya Str. 7, Sochi, 354008, Russia
}

vss2000@mail.ru

\begin{abstract}
The problem of system design of complex technical objects based on intelligent technologies is considered. An optimization model for the conceptual design of a micro-minisatellite based on a genetic algorithm is discussed. An artificial neural network model of a propulsion system is considered, as well as a heuristic algorithm for analyzing the crosscorrelation of telemetric parameters of a micro-mini-satellite. The concept of constructing an intelligent system for information support of the life cycle of a complex technical object based on the considered models and algorithms is proposed.
\end{abstract}

\section{Introduction}

One of the directions for improving the life cycle management processes of complex technical objects is the transition to contracts for the full product life cycle. An objective need in recent years has been the optimization of the relationship between the customer and the manufacturer, focused on supporting the operation of complex technical object, increasing the degree of readiness and achieving the specified tactical and technical characteristics. This approach is implemented in the form of the concept of Performance-Based Lifecycle Product Support (CoPBL) [1, 2]. According to this approach, the manufacturer receives comprehensive access to product life cycle information during the operation stage.

In our case, as an example of a complex technical object, we consider a micro-mini-satellite. Currently, micro-mini-satellites are widely used for scientific, commercial and other purposes, in particular, remote sensing of the earth, environmental monitoring, communications, and scientific experiments [3-7].

One of the unresolved problems in development of information support systems for the complex technical object life cycle is the efficient use of a large amount of data appearing at various stages of the life cycle in order to optimize the operation stage [8-10].

The expansion of duration of the life cycle of complex technical object and its technical and economic efficiency can be based on the intelligent information support with using the integrated life cycle models. The construction of end-to-end models of the object life cycle becomes possible due to big data processing technologies that integrate distributed data and make it possible to increase the efficiency of using the above-mentioned components of the information support system [11-13].

The components of the micro-mini-satellite life cycle information support system focused on the use of big data technologies [14-17]: 
1) Micro-mini-satellite model for design optimization based on the genetic algorithm,

2) Artificial neural network model of the micro-mini-satellite propulsion system, which allows estimating the remaining fuel supply and technical condition under conditions of uncertainty,

3) Heuristic algorithm for analyzing the cross-correlation of telemetric parameters of a micro-minisatellite.

Some elements of this approach were applied for design of educational micro-mini-satellite "USATUSAT".

\section{Micro-mini-satellite and Data Acquisition}

The micro-mini-satellite can be included into the class of complex technical systems. In the structure of micro-mini-satellite there are remote sensing system and service units that provide the process of object functioning. The generalized scheme of the micro-mini-satellite is presented in figure 1.

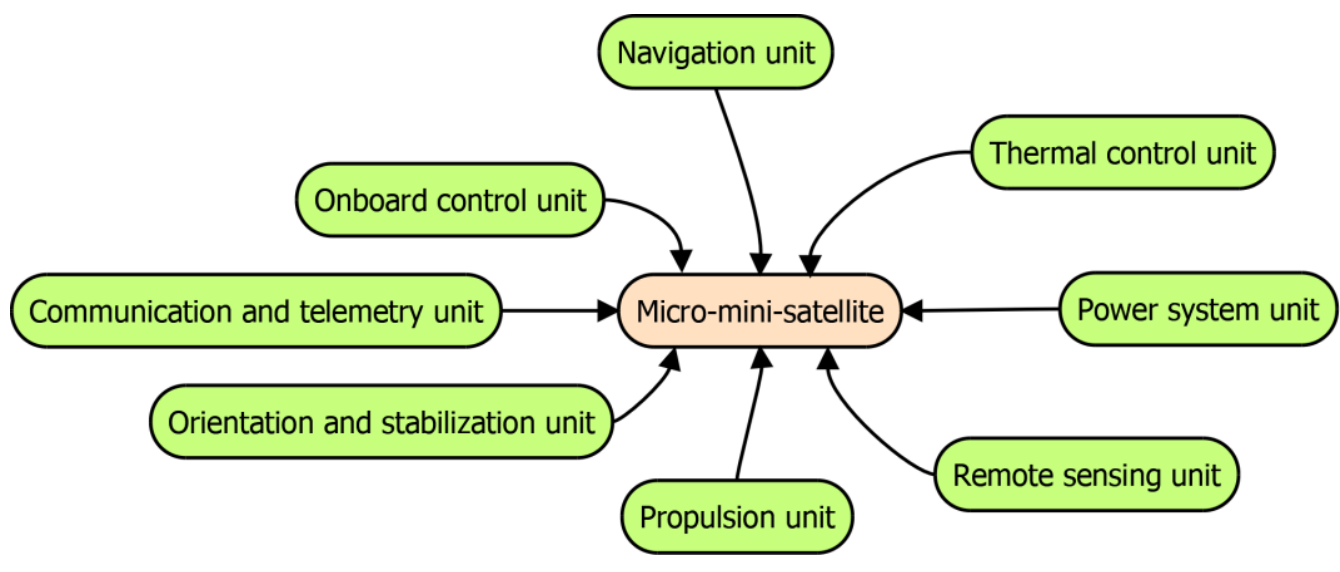

Figure 1. Main units of micro-mini-satellite.

The requirements for high reliability and functionality of the on-board satellite complex are related to the difficult flight situation and hard real-time conditions during the launch and operation $[18,19]$.

The process of accumulation of information at the pre-operational stages of the micro-mini-satellite life cycle is shown in figure 2 .

Level of Life Sycle

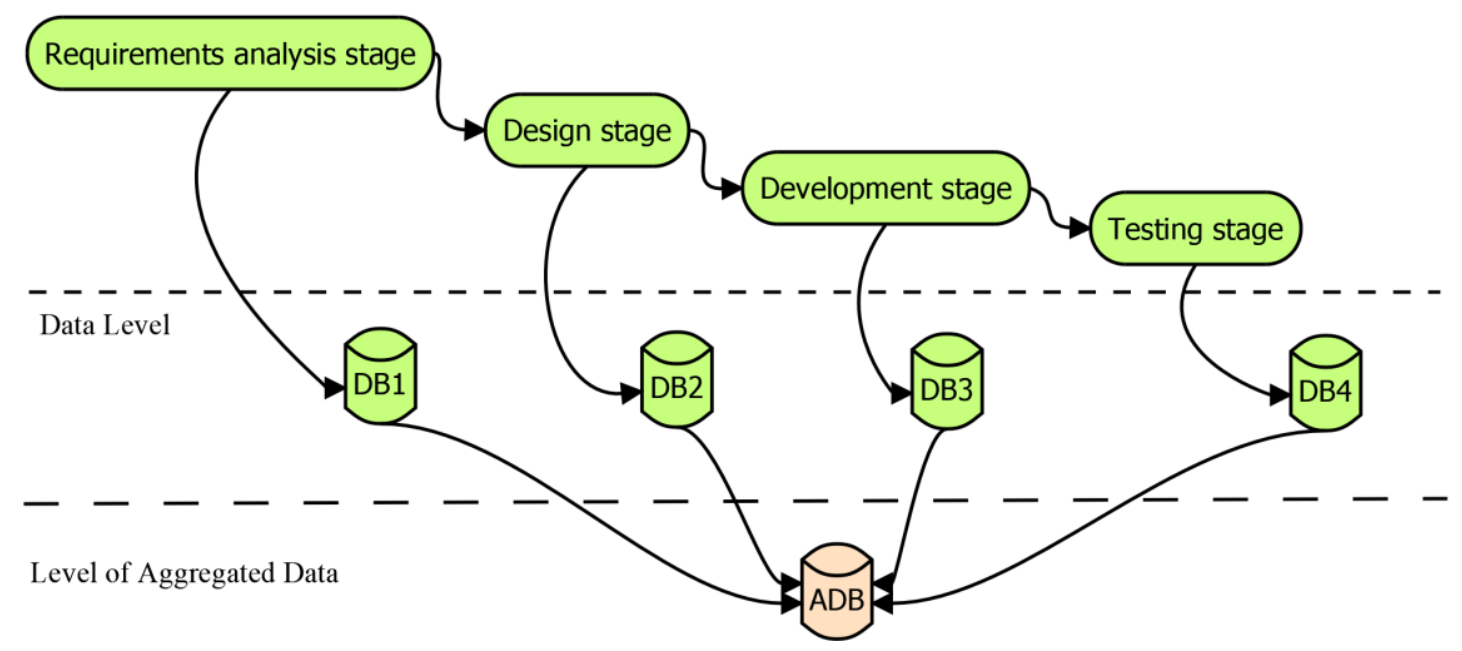

Figure 2. The process of accumulation of information at the pre-operational stages. 
To support decision-making during satellite operation, additional information is required on the previous life cycle stages, i.e. design, manufacturing, and testing. Data can be located in distributed storages and relate to the area of responsibility of various organizations that are participants in the life cycle.

At various stages of the micro-mini-satellite life cycle, it becomes necessary to process and store large amounts of information, which can be further used to increase the efficiency of flight control and perform objective operations. In figure 2 three levels are presented: level of life cycle processes, level of data generation, data transition and storage in local databases; level of aggregation of entire data into an Aggregate Database.

\section{System design and genetic algorithms}

At the stage of system design, in order to make a decision of architecture selection for the object, it is necessary to create many alternative versions of the product $V_{j}, j=\overline{1, J}$, contained in the product database of the product data management system (PDM).

Basic characteristics of design optimization are various constraints $g_{i}, i=\overline{1, I}$ of mass, dimensions, cost, power consumption, reliability, accuracy and others. These constraints are represented as the required ranges of values for operation units of the corresponding level $G_{i}^{r e q}$ of system architecture. For example, micro-mini-satellite requirements are stored at the top level of the product database, and subsystem requirements are stored at the second level, etc.

The compatibility conditions for product components are specified in the form of set-theoretic functions $U_{i}, i=\overline{1, I_{U}}$. Variable parameters are the characteristics of the lower level elements $\bar{X}=\left\{x_{1}, \ldots, x_{m}, \ldots x_{M}\right\}$. The architecture selection is based on the assessment of alternatives according to a variety of criteria $K_{1}, \ldots, K_{N} \rightarrow \max$ that determine the objective functioning of the micro-mini-satellite.

The task of optimizing the design parameters of a complex technical object on a set of constraints is an NP-complex mixed integer multicriteria nonlinear programming problem. To solve such tasks, it is possible to use optimization genetic algorithms. In terms of the genetic algorithm, the micro-minisatellite version is an individual; the components and subsystems of the product (for example, orientation and stabilization system, propulsion system and others) are chromosomes; the characteristics and attributes of product components are genes. The result of the genetic operators of crossing and mutation are new products that are individuals with improved characteristics in accordance with given constraints.

The characteristics or genes of the lower level can be numerical, interval or multiple values. The groups of such characteristics are denoted, respectively, by $X^{n}, X^{i}$, and $X^{\mathrm{m}}$. Set of genes or characteristics is denoted by $\bar{X}=X^{\mathrm{n}} \cup X^{\mathrm{i}} \cup X^{\mathrm{m}}=\left\{x_{1}, \ldots, x_{m}, \ldots x_{M}\right\}$. Then, by nonlinear convolution, the multicriteria problem $K_{1}, \ldots K_{N} \rightarrow \max$ reduces to a single criterion:

$$
\begin{gathered}
F\left(K_{1}, \ldots K_{N}\right) \rightarrow \text { extr } \\
\left\{\begin{array}{c}
g_{i}\left(x_{1}, \ldots, x_{M}\right) \in G_{i}^{r e q}, i=\overline{1, I} \mid U_{i}, i=\overline{1, I_{U}} \\
\bar{X}=X^{\mathrm{n}} \cup X^{\mathrm{i}} \cup X^{\mathrm{m}}=\left\{x_{1}, \ldots, x_{m}, \ldots x_{M}\right\}
\end{array},\right.
\end{gathered}
$$

where $F\left(K_{1}, \ldots, K_{N}\right)$ is fitness function; $\bar{X}=\left\{x_{1}, \ldots, x_{m}, \ldots x_{M}\right\}$ are variable characteristics or lower level genes; $g_{i}\left(x_{1}, \ldots, x_{M}\right)$ are technical and economic constraints on the micro-mini-satellite architecture; $G_{i}^{\text {req }}$ are required ranges of constraint values $g_{i} ; U_{i}, i=\overline{1, I_{U}}$ are set-theoretic functions describing the compatibility conditions of product components. 
Thus, the genetic algorithm allows us to explore many options for the architecture selection of a complex technical object and choose the optimum for a given set of criteria. When forming the system architecture of a technical object, a large amount of reliable information is required. It is not always possible to measure a number of important parameters in the system, for example, fuel consumption. Consumption is associated with solving flight problems under the influence of various uncertainty factors. When selecting architecture of a satellite, this factor must be taken into account.

\section{Neural network model of the micro-mini-satellite propulsion system}

At present, micro-mini-satellites are equipped with micro propulsion systems. The design and operation of micro-mini-satellites and micro-engines is carried out in conditions of significant uncertainty.

Given the current level of development of measurement technology, it is impossible to place fuel and thrust sensors on board a satellite, and the calculation method does not satisfy the accuracy requirements.

To estimate the fuel supply and the technical condition of the micro-mini-engine based on the operational data stream, we can apply a model based on a complex of artificial neural networks and a logic unit. The developed artificial neural network model includes three neural networks $\mathrm{ANN}_{1}$, $\mathrm{ANN}_{2}$, and $\mathrm{ANN}_{3}$. All developed artificial neural networks are multilayer perceptrons trained by the method of back propagation of error. As a training sample, we used the calculated and experimental data obtained at the design and testing stages of the micro propulsion systems. The input $X$ is fed to the propulsion systems for the obtaining of thrust, and the output $Y$ allows to evaluate the amount of thrust.

The set of neural networks based on $\mathrm{ANN}_{1}, \mathrm{ANN}_{2}$, and $\mathrm{ANN}_{3}$ is shown in figure 3. The sets $X^{*}$ and $Y^{*}$ are output parameters of $\mathrm{ANN}_{1}$ and $\mathrm{ANN}_{2}$.

The $\mathrm{ANN}_{1}$ neural network (with a structure of 12-5-3-3) is designed to determine the dependence of the output information $Y^{*}$ on the input $X$ and is used in the simulation mode of the operation of the micro-mini-satellite remote control.

The $\mathrm{ANN}_{2}$ neural network (with a structure of 3-3-5-12) solves the inverse problem and determines the dependence of the input information $X^{*}$ on the output $Y$. The results of the operation of the ANN2 neural network are used in the monitoring mode to solve the problem of diagnosing the technical condition of the micro-mini-satellite propulsion system.

The $\mathrm{ANN}_{3}$ neural network (with a structure of 15-6-4-2) is used to determine the fuel consumption $G_{\tau}$ and assess the technical condition $S_{\tau}$. In $\mathrm{ANN}_{3}$, the data streams $X$ and $Y$ are used as input.

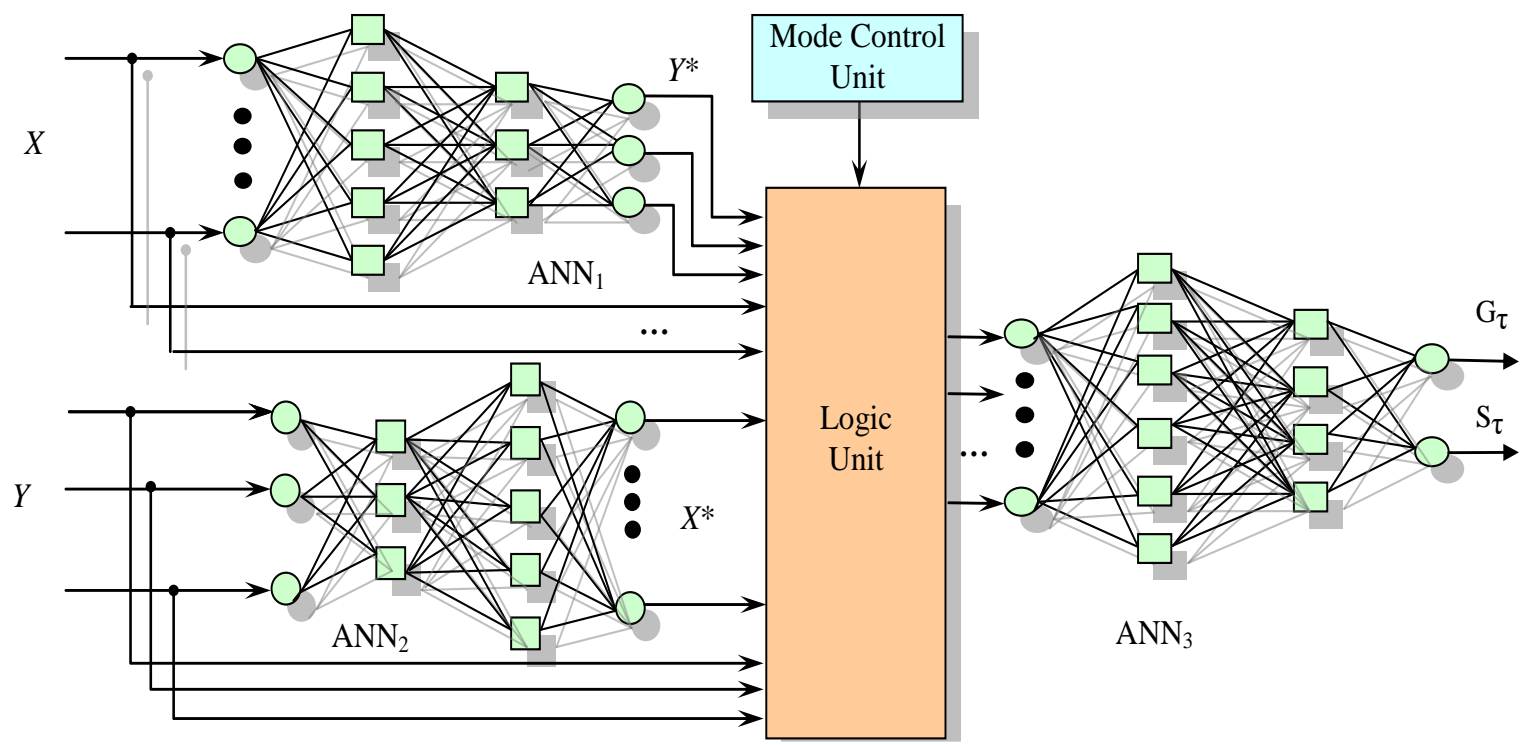

Figure 3. The complex of neural networks based on $\mathrm{ANN}_{1}, \mathrm{ANN}_{2}$ and $\mathrm{ANN}_{3}$. 
The fuel consumption data acquired in the process of testing the remote control allow increasing the accuracy and reliability of the training sample.

In the mode of modeling the operation of the propulsion system of the micro-mini-satellite, the logic block provides the interaction of $\mathrm{ANN}_{1}$ and $\mathrm{ANN}_{3}$. In the monitoring mode interaction of $\mathrm{ANN}_{2}$ and $\mathrm{ANN}_{3}$ also improves the accuracy of estimating fuel consumption $G_{\tau}$ using additional information about the technical condition $S_{\tau}$ of the propulsion system.

The design of a neural network algorithm using the set of neural networks is based on the analysis of a large array of data. The data are accumulated at various stages of the life cycle of the microengine and micro-mini-satellite, which can be located on distributed storages.

\section{Heuristic algorithm for analyzing the cross-correlation of telemetry parameters of a micro- mini-satellite}

During flight tests and operation of the micro-mini-satellite, a significant amount of telemetric information is accumulated. On the base of this information, in the conditions of the mission control center operation, it is possible to obtain a forecast of the remaining life of the micro-mini-satellite. Also, we can detect hidden gradual failures by analyzing the structural and functional interaction of the micro-mini-satellite subsystems.

The technical complexity of the micro-mini-satellite design gives rise to complex structural and functional relationships between the technical parameters of the micro-mini-satellite subsystems, which can be complexly correlated. The analysis of the relationships between the telemetric parameters of the micro-mini-satellite is a complex scientific and technical task, which in the conditions of a large flow of information can be solved by developing intelligent algorithms for analytical data processing.

To assess the dynamics of indicators of the technical state of the micro-mini-satellite, it is proposed to use the analysis of the cross-correlation of telemetric parameters of the main subsystems of the micro-mini-satellite. The reason of the cross-correlation of telemetric parameters is determined by the design features, the mechanisms of interaction of the micro-mini-satellite subsystems and the architecture of the on-board system.

The regression analysis of the telemetric data set $X=\left\{x_{1}(t), \ldots x_{i}(t), \ldots x_{j}(t), \ldots x_{N}(t)\right\}$, where $x_{i}(t)$ is the pulse discrete signal of $i$-th telemetric parameter, is difficult due to its large dimension, as well as the diversity and complexity of the relationship between telemetric parameters. In addition, a number of telemetry parameters have time delay relative to each other with a random time lag $\tau$ (crosscorrelation radius). Thus, the direct application of regression and correlation analysis tools is difficult. Therefore, instead of signals $x_{i}(t)$ it is proposed to use peak-discrete function $\tilde{x}_{i}(t)$ :

$$
\tilde{x}_{i}(t)=\left\{\begin{array}{l}
\operatorname{sign}\left(\frac{\Delta x_{i}\left(t_{k-1}\right)}{\Delta t}\right), \text { if } \frac{\Delta x_{i}\left(t_{k-1}\right)}{\Delta t} \frac{\Delta x_{i}\left(t_{k}\right)}{\Delta t}<0, k=0,1, \ldots \\
0, \text { otherwise }
\end{array}\right.
$$

An example of a peak-discrete function $\tilde{x}_{i}(t)$ is shown in figure 4 .

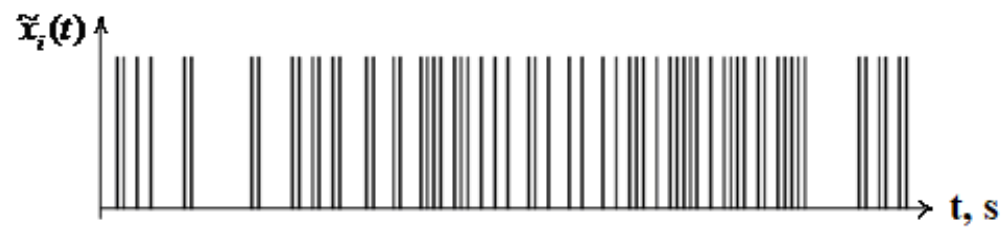

Figure 4. Peak-discrete function $\tilde{x}_{i}(t)$. 
Then, instead of calculating the cross-correlation function of continuous signals, which, given the difference in the shape of the signals $x_{i}(t)$ and $x_{j}(t)$, gives an unsatisfactory result, the crosscorrelation function of peak-discrete signals $\tilde{x}_{i}(t)$ and $\tilde{x}_{j}(t)$ :

$$
B_{i, j}(\tau)=\frac{1}{K} \sum_{t=0}^{K} \tilde{x}_{i}(t) \tilde{x}_{j}(t-\tau)
$$

An analysis of the dynamics of the correlation function $B_{i, j}(\tau)$ together with an estimate of the expected value $M_{i, j}(\tau)$ and variance $\sigma_{i, j}^{2}(\tau)$ of the cross-correlation of signals gives a developed representation of the cross correlation between the telemetry parameters $i$ and $j$.

As part of the study (analysis), an algorithm and a software module for the analytical processing of telemetry data of the micro-mini-satellite are proposed, which performs the following functions:

- detection of cross-correlation between tele metric parameters;

- determination of the type of correlation, namely instant or with a random time lag;

- analysis and control of statistical indicators of cross-correlation $B_{i, j}(\tau), M_{i, j}(\tau)$, and $\sigma_{i, j}^{2}(\tau)$.

\section{Architecture of information support system}

Improving the efficiency of operation and objective use of the micro-mini-satellite requires the development of an integrated hardware and software complex for information support of the micromini-satellite life cycle as part of the Mission Control Center.

The scheme of the information support system is presented in figure 5 .

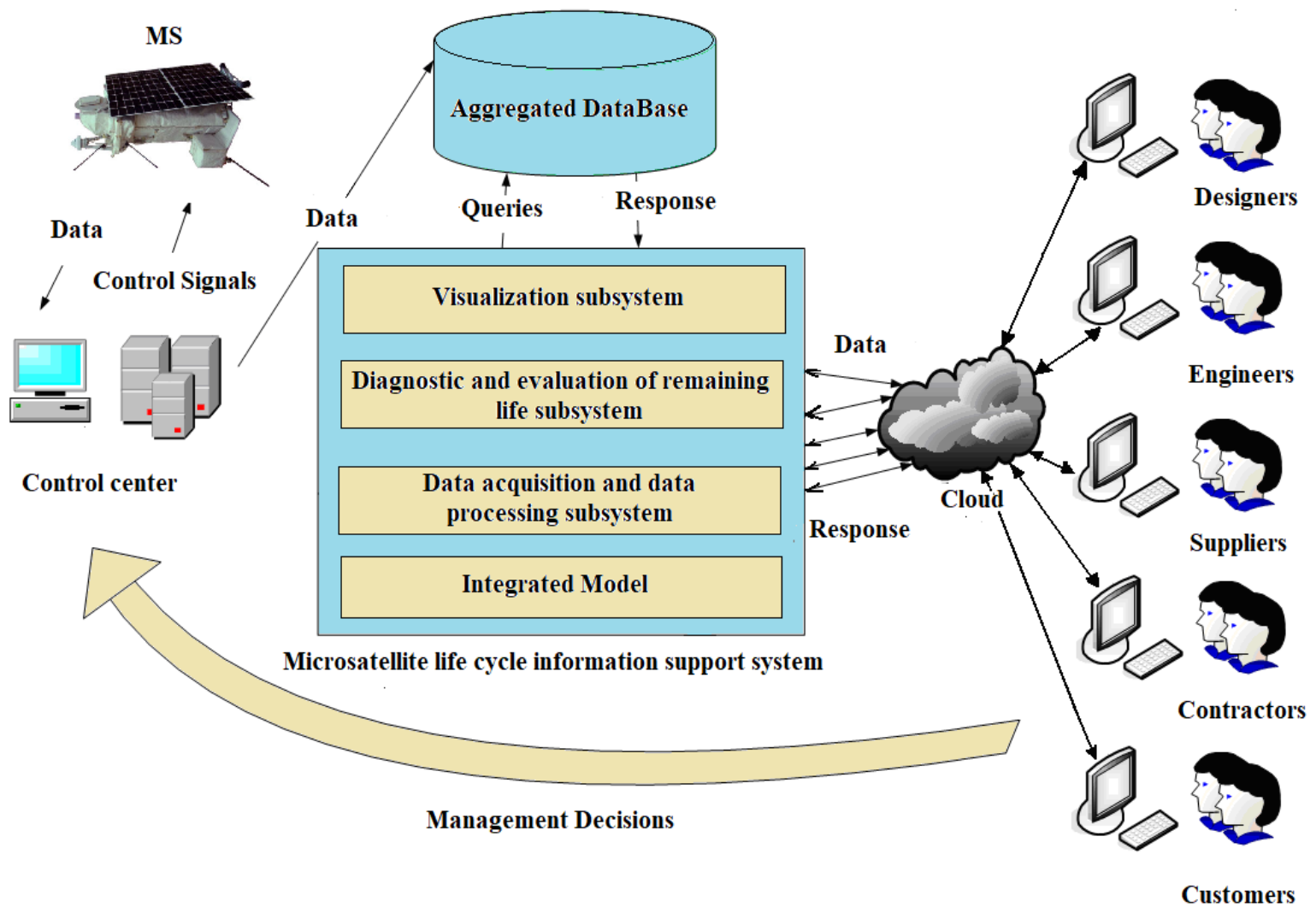

Figure 5. The scheme of the information support system during operation. 
The information support system of the life cycle of the micro-mini-satellite includes the following subsystems: information collection and processing subsystem; subsystem of three-dimensional modeling and visualization; subsystem for assessing the resources and state of the micro-mini-satellite; a complex of integrated life cycle models of a complex technical object.

The information support system is intended both for use by flight control center employees and territorially remote technical experts and users of aerospace information in a distributed mode.

To solve the problems of information support for the micro-mini-satellite life cycle, a set of computer models has been created, including 3D models of the micro-mini-satellite design and its control systems, information models of the production process, virtual 3D models of satellite operation processes, models of logistics supply chains in the interaction of designers, manufacturers, micromini-satellite operators and consumers of objective information.

The aggregated database of the micro-mini-satellite life cycle information support system contains hybrid heterogeneous information replenished from distributed information systems, namely design, production, technological, testing and operational data (figure 2).

In particular, operational telemetry information about micro-mini-satellite flight contains measurements of a large number of parameters over long time intervals. The volume of telemetric information can be significant, and the rate of data feed into the aggregated database is relatively high. Together, these factors allow talking about the applicability of Big Data technology for processing large amounts of data as part of a micro-mini-satellite life cycle information support system.

\section{Conclusion}

The main goal of the conceptual system design of complex technical objects is improving the life cycle management processes. It becomes possible on the basis of intelligent methods and integrated information support.

The principles underlying the information support of the micro-mini-satellite life cycle make it possible to generalize the proposed models and methods for managing the life cycle of a complex technical object.

An integral part of the life cycle information support system of a complex technical object should be a complex of integrated life cycle models of a complex technical object, built on the basis of intelligent technologies.

The information support system for the micro-mini-satellite life cycle directly uses the following elements of the complex of models: trained neural networks with switching logic, virtual and visual models of a complex technical object, heuristic models and methods for analyzing telemetric data.

\section{References}

[1] Geary S, Koster S, Randall W and Haynie J 2010 Performance-based life cycle product support strategies: enablers for more effective government participation Defense Acquisition Review J. October

[2] Armenakis A A, Harris S G, Cole M S, Fillmer J L and Self D R 2007 A top management team's reactions to organizational transformation: The diagnostic benefits of five key change sentiments J. of Change Management 7 pp 273-290

[3] Sweeting M N 2018 Modern small satellites-changing the economics of space Proceedings of the IEEE 1063 pp 343-361.

[4] Sweeting M N 2000 Micro-mini-satellites for affordable access to space Miau JJ., Holdaway R. (eds) Reducing the Cost of Spacecraft Ground Systems and Operations. Space Technology Proceedings 3. Springer, Dordrecht

[5] Blinov V N et al 2015 Design features and research of electrothermal microthrusters with autonomous heating elements for the purposes of small space vehicle orbital manoeuvring Indian Journal of Science and Technology 8(27) IPL0581

[6] Blinov V N et al 2015 The studies of small space vehicles ammoniac electrothermal engine units design and structural layout Modern Applied Science 9 no 5

[7] Yan Z et al 2013 System dynamic characteristic simulation of spacecraft propulsion system based 
on AMESim Advanced Materials Research 605-607 pp 679-683

[8] Volkov A, Kopyrin A, Kondratyeva N and Valeev S 2019 Multilevel data acquisition system of energy losses in recreation areas Twelfth Int. Conf. Management of large-scale system development (MLSD) (Moscow, Russia) pp 1-4

[9] Blinov V N, Valeev S S, Kondratyeva N V, Karimov R R, Kovtunenko A S and Kuzmina E A 2019 Supporting the life cycle of complex technical systems on the basis of intelligent technologies and predictive analytics CEUR Workshop Proc.: Data Science Session at the 5th Int. Conf. on Information Technology and Nanotechnology DS-ITNT 2019 (Samara, Russian Federation, 21-24 May 2019) 2416 pp 314-323

[10] Valeev S S, Kondratyeva N V, Kovtunenko A S, Timirov M A and Karimov R R 2019 Distributed stream data processing system in multi-agent safety system of infrastructure objects CEUR Workshop Proc.: Data Science Session at the 5th Int. Conf. on Information Technology and Nanotechnology DS-ITNT 2019 (Samara, Russian Federation, 21-24 May 2019) 2416 pp 324-331

[11] Bychkov I, Oparin G, Tchernykh A, Feoktistov A, Bogdanova V and Gorsky S 2017

Conceptual Model of Problem-Oriented Heterogeneous Distributed Computing Environment with Multi-Agent Management Procedia Computer Science 103 162-167

[12] Bychkov I V, Oparin G A, Bogdanova V G, Pashinin A A and Gorsky S A 2017 Automation development framework of scalable scientific web applications based on subject domain knowledge Parallel Computing Technologies ed V Malyshkin (Springer: Cham) LNCS 10421 pp 278-288

[13] Kondratyeva N V and Valeev S S 2016 Simulation of the life cycle of a complex technical object within the concept of Big Data CEUR Proceedings of $3^{\text {rd }}$ Russian Conference Mathematical Modeling and Information Technologies pp 216-223

[14] Kondratyeva N, Valeev S 2016 Fatigue test optimization for complex technical system on the basis of lifecycle modeling and big data concept Proc. of 10th Conf. on Application of Information and Communication Technologies AICT2016 pp 1-4

[15] Carroll D L, Cardin J M, Burton R L, Benavides G F 2015 Propulsion unit for CUBESATS (PUC) 62nd JANNAF Propulsion Meeting (7th Spacecraft Propulsion) http://www.cuaerospace.com/Portals/SiteContent/Publications/2015/ Carroll_JANNAF_2015_PUC_4059.pdf

[16] Vasilyev V I, Valeyev S S and Shilonosov A A 2001 Design of neurocontroller for gas-turbine engine multi-mode control Proc. 8th Int. Conf. on Neural Information Processing ICONIP-2001

[17] Karimov R R, Islamgulov T V and Karpenko O Yu 2012 Application of neural network and genetic algorithms to life cycle information support of complex technical object Proc. of 14th Int. Workshop on Computer Science and Information Technologies CSIT'2012 1 pp 228-231

[18] Curry D M, Beaver W W and Dagli C H 2018 A system-of-systems approach to improving intelligent predictions and decisions in a time-series environment SoSE 2018 pp 98-105

[19] Gosavi A, Agarwal S and Dagli C H 2017 Predicting response of risk-seeking systems during project negotiations in a system of systems IEEE Systems $J .113$ pp 1557-1566 